\title{
Higher-Order Semi-Implicit Projection Methods
}

\author{
M. L. Minion
}

This article was submitted to

Numerical Simulations of Incompressible Flows

Half Moon Bay, CA, June 19-21, 2001

U.S. Department of Energy

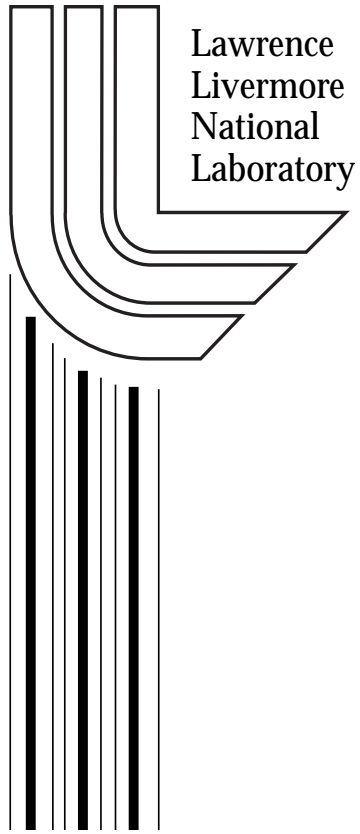

September 6, 2001 


\section{DISCLAIMER}

This document was prepared as an account of work sponsored by an agency of the United States Government. Neither the United States Government nor the University of California nor any of their employees, makes any warranty, express or implied, or assumes any legal liability or responsibility for the accuracy, completeness, or usefulness of any information, apparatus, product, or process disclosed, or represents that its use would not infringe privately owned rights. Reference herein to any specific commercial product, process, or service by trade name, trademark, manufacturer, or otherwise, does not necessarily constitute or imply its endorsement, recommendation, or favoring by the United States Government or the University of California. The views and opinions of authors expressed herein do not necessarily state or reflect those of the United States Government or the University of California, and shall not be used for advertising or product endorsement purposes.

This is a preprint of a paper intended for publication in a journal or proceedings. Since changes may be made before publication, this preprint is made available with the understanding that it will not be cited or reproduced without the permission of the author.

This report has been reproduced

directly from the best available copy.

Available to DOE and DOE contractors from the

Office of Scientific and Technical Information

P.O. Box 62, Oak Ridge, TN 37831

Prices available from (423) 576-8401

http://apollo.osti.gov/bridge/

Available to the public from the National Technical Information Service

U.S. Department of Commerce 5285 Port Royal Rd., Springfield, VA 22161 http://www.ntis.gov/

OR

Lawrence Livermore National Laboratory Technical Information Department's Digital Library http://www.llnl.gov/tid/Library.html 


\begin{abstract}
A semi-implicit form of the method of spectral deferred corrections is applied to the solution of the incompressible Navier-Stokes equations. A methodology for constructing semi-implicit projection methods with arbitrarily high order of temporal accuracy in both the velocity and pressure is presented. Three variations of projection methods are discussed which differ in the manner in which the auxiliary velocity and the pressure are calculated. The presentation will make clear that projection methods in general need not be viewed as fractional step methods as is often the practice. Two simple numerical examples are used to demonstrate fourth-order accuracy in time for an implementation of each variation of projection method.
\end{abstract}




\section{Introduction}

A great deal of effort has been dedicated to the design of numerical methods for the simulation of high Reynolds number flow, often including techniques for incorporating turbulence models to represent sub-grid scale effects. There are, however, many situations of interest in which the Reynolds number is "moderate", i.e. high enough so that the Stokes equations are not a reasonable model, but low enough so that the flow can be resolved on a computational grid and no subgrid scale model is needed. The modeling of biological systems such as the flow of blood in the heart or the swimming of organisms is just one such example. Often in these examples, it is desirable to compute the motion of the fluid with high accuracy since other components in the system which depend on the fluid are of interest (e.g. the motion of membranes or the passive transport of solutes). As the interest in numerical modeling in many fields in the applied sciences and the speed and memory capacity of computers continue to increase, the need for accurate, efficient, and adaptable numerical methods for moderately viscous flows should increase.

Numerical methods for viscous flows must treat viscous terms implicitly in order to avoid a severe time step restriction. This paper introduces semi-implicit projection methods with arbitrarily high temporal accuracy for the integration of the equations governing viscous incompressible flows. Furthermore, the methods require the solution of only two linear implicit equations for which efficient high-order solvers exist. Therefore the use of the current methods with complex geometry as well as spatial and temporal adaptivity is possible immediately.

The projection methods in this paper are based on a method of lines approach coupled with a semi-implicit method for integrating ordinary differential equations (ODEs) based on the method of spectral deferred corrections (SDC) [DGR00, Min01]. These so-called SISDC methods have several advantages over the more traditional semi-implicit multi-step and Runge-Kutta methods. Higher-order semiimplicit linear multi-step methods are not self starting, they typically require a severely restricted time step for stability, and they present difficulties when variable time stepping is required (see e.g. [ARW95, FHV97]). On the other hand, semi-implicit Runge-Kutta

methods are self starting and generally have good stability properties, 
and efficient methods for orders up to five have been proposed. (See e.g. [SZ96, ARS97, KC01, CdFN01]). It has been well documented however, that when combined with a method of lines approach for PDEs, Runge-Kutta methods typically suffer from a loss of accuracy when time dependent boundary conditions are prescribed, unless special care is taken when imposing intermediate boundary conditions [SSVH86, CGAD95]. Suggestions for restoring full accuracy in certain cases have been proposed (e.g. [AGC96, Pat97, CP01]), but at present, no general strategy for semi-implicit Runge-Kutta methods has been developed.

The SDC method has the advantage that a relatively simple numerical method is used to compute a higher-order solution. This is accomplished by using the simple method to solve a series of correction equations during each time step, each of which increases the order of accuracy of the solution. This makes SISDC methods particularly attractive to problems possessing disparate time scales since a time-split approach can be used without being limited to lower-order accuracy. The method of spectral deferred corrections has been used already in this manner in the context of unsteady combustion [HRZ98, HRSZ99]. Furthermore, imposing correct boundary conditions for a semi-implicit method of lines approach for PDEs, although certainly not trivial, is more transparent for SISDC methods because of the simplicity of the underlying method. An overview of SISDC methods is presented in Sect. 3 .

The strategy for the construction of SISDC projection methods relies on applying the method of lines approach to a non-standard form of the equations of motion. The connection between this form and projection methods is discussed in Sect. 2. Three variations of a generalized projection method will be presented in Sect. 4. Numerical examples set in an idealized geometry are presented in Sect. 5. Fourthorder convergence for both pressure and velocity is demonstrated in these examples.

\section{The Equations for Incompressible Flow}

In this Section, different formulations of the equations of motion of an incompressible fluid are discussed. In an $N$-dimensional bounded domain $\Omega$, the usual form of the equations of motion are given by 
the incompressible Navier-Stokes equations in terms of the velocity $\mathbf{u}$ and the pressure $p$. Denoting the kinematic viscosity by $\nu$, the Navier-Stokes equations are

$$
\begin{aligned}
\mathbf{u}_{t}+\nabla p & =-(\mathbf{u} \cdot \nabla) \mathbf{u}+\nu \nabla^{2} \mathbf{u} \\
\nabla \cdot \mathbf{u} & =0
\end{aligned}
$$

with boundary conditions

$$
\left.\mathbf{u}\right|_{\partial \Omega}=\mathbf{u}_{b}
$$

Numerous equivalent formulations of these equations have been developed to facilitate their numerical approximation.

Projection methods first introduced by Chorin [Cho68, Cho69] are based on the observation that the left-hand side of Eq. (1) is a Hodge decomposition. Hence an equivalent projection formulation is given by

$$
\mathbf{u}_{t}=\mathbf{P}\left[-(\mathbf{u} \cdot \nabla) \mathbf{u}-\nabla p+\nu \nabla^{2} \mathbf{u}\right]
$$

where $\mathbf{P}$ is the operator which projects a vector field onto the space of divergence-free vector fields with appropriate boundary conditions. In general, the strategy employed for one time step in a generic projection method is to first approximate Eq. (1) without regard to the divergence constraint (2) to yield an "auxiliary velocity" $\mathbf{u}^{*}$, and then to project $\mathbf{u}^{*}$ onto the space of divergence-free fields to give the new velocity. For this reason, projection methods are often referred to as fractional step methods.

Second order, semi-implicit projection methods in which the advective terms in the equation for $\mathbf{u}^{*}$ are handled explicitly while the viscous terms are handled implicitly have been developed in [KM85, BCG89] and others. The most significant issue that must be resolved in these methods is the imposition of boundary conditions for $\mathbf{u}^{*}$ in the implicit step. Since the projection of $\mathbf{u}^{*}$ is usually implemented by the solution of a Poisson problem for which only one boundary condition can be prescribed, the boundary conditions for $\mathbf{u}^{*}$ must be chosen in such a way that the remaining velocity boundary conditions are satisfied. The boundary conditions on $\mathbf{u}^{*}$ also effect the accuracy of the pressure gradient. An overview as well as a further analysis of boundary conditions for second-order semi-implicit projection methods appears in [BCM01]. 
In order to develop higher-order numerical methods based on the projection method strategy, it is helpful to consider an alternative formulation of the incompressible Navier-Stokes equations based on an auxiliary variable equal to the fluid velocity plus the gradient of a scalar. Such formulations were first introduced by Oseledets [Ose89] and many variations have since been proposed for use in numerical methods for various problems [But93, Cor95, Cor96, EL96, EL97, RR98, SC96, CM00].

Consider two new variables, $\mathbf{m}$ and $\chi$ which are related to the fluid velocity by

$$
\mathbf{m}=\mathbf{u}+\nabla \chi .
$$

The vector field $\mathbf{m}$ and the potential $\chi$ can be chosen to satisfy evolution equations in such a way that the fluid velocity and pressure derived from them satisfy the Navier-Stokes equations. One possibility which facilitates the development of accurate projection methods is

$$
\begin{aligned}
\mathbf{m}_{t}+(\mathbf{u} \cdot \nabla) \mathbf{u} & =-\nabla q+\nu \nabla^{2} \mathbf{m} \\
\left.\mathbf{u}\right|_{\partial \Omega} & =\mathbf{u}_{b} .
\end{aligned}
$$

where $\nabla q$ is an arbitrary gradient that will be discussed later and

$$
\mathbf{u}=\mathbf{P}(\mathbf{m}) .
$$

In this formulation, the pressure has been eliminated from the equations; however, it can be recovered from the potential $\chi$ by enforcing the equivalence of equations (1) and (6) giving

$$
p=q+\chi_{t}-\nu \nabla^{2} \chi
$$

Initially, $\nabla \chi$ can be zero, (i.e. $\mathbf{m}=\mathbf{u}$ ), or $\nabla \chi$ can be non-zero so long as the choice is consistent with Eq. (5). Note that the boundary conditions are given in terms of $\mathbf{u}$, which by Eq. (5), implies that there is a coupling of the boundary conditions of $\mathbf{m}$ and $\nabla \chi$ (See [BCM01]).

Clearly if $\nabla q$ is defined as the pressure gradient and $\mathbf{m}$ is given initial and boundary values equal to $\mathbf{u}$, then Eq. (6) reduces to Eq. (1). If $\nabla q$ is identically zero, the resulting equations are equivalent to those used as the basis of the numerical method in [EL96]. As discussed in the next section, the key observation to make is that a single step of a projection method can be thought of as approximating Eq. (6) rather than Eq. (1). 


\section{Semi-Implicit Spectral Deferred Cor- rections}

In [DGR00], the SDC method for the solution of ODEs is introduced. The strategy within each time step of the method is to use a simple numerical method (specifically forward or backward Euler) to calculate a provisional solution at specific points within the interval of the time step. Then, the same simple method is used to solve a series of correction equations each of which increases by one the order of accuracy of the provisional solution. The correction equation is cast in a form similar to the Picard integral iteration, hence the points at which the provisional solution and the corrections are calculated correspond to quadrature nodes. A semi-implicit version of the SDC method (SISDC) suitable for use with the Navier-Stokes equations will be briefly described below. For more complete details see [DGR00] and [Min01].

Consider the ODE

$$
\begin{aligned}
& \phi^{\prime}(t)=F(t, \phi(t))=F_{E}(t, \phi(t))+F_{I}(t, \phi(t)) \quad t \in[a, b] \\
& \phi(a)=\phi_{a}
\end{aligned}
$$

where $F_{E}$ is a non-stiff term that will be handled explicitly, and $F_{I}$ is a stiff term that will be handled implicitly.

For a given time step on the interval $\left[t_{n}, t_{n+1}\right]$, the first step in the SISDC method is to compute a provisional solution $\tilde{\phi}\left(t_{m}\right)$ at the points $t_{m}$ for $m=0 \ldots p$ with $t_{n}=t_{0}<t_{1}<\ldots<t_{p}=t_{n+1}$. Assuming that $\phi\left(t_{n}\right)$ is known and using the notation $\tilde{\phi}\left(t_{m}\right) \equiv \tilde{\phi}^{m}$, a first-order, semi-implicit numerical method for computing $\tilde{\phi}$ is

$$
\tilde{\phi}^{m+1}=\tilde{\phi}^{m}+\Delta t_{m}\left[F_{E}\left(t_{m}, \tilde{\phi}^{m}\right)+F_{I}\left(t_{m+1}, \tilde{\phi}^{m+1}\right)\right],
$$

where $\Delta t_{m}=t_{m+1}-t_{m}$ and $\tilde{\phi}^{0}=\tilde{\phi}\left(t_{n}\right)$. For clarity, the interval $\left[t_{m}, t_{m+1}\right]$ will be referred to as a substep as opposed to the time step $\left[t_{n}, t_{n+1}\right]$.

Following [DGR00], if one regards $\tilde{\phi}$ as a continuous function, a measure of the error in the provisional solution can be written

$$
E(t, \tilde{\phi})=\tilde{\phi}_{0}+\int_{t_{0}}^{t} F(\tau, \tilde{\phi}(\tau)) d \tau-\tilde{\phi}(t)
$$


Defining the correction to the provisional solution $\delta(t)$ by $\phi(t)=\tilde{\phi}(t)+$ $\delta(t)$, some elementary algebra yields

$$
\delta(t)=\int_{t_{0}}^{t} F(\tau, \tilde{\phi}(\tau)+\delta(\tau))-F(\tau, \tilde{\phi}(\tau)) d \tau+E(t, \tilde{\phi}) .
$$

The SISDC method proceeds by computing a sequence of corrections $\delta^{k}(t)$, where each $\delta^{k}$ is computed by first approximating $E(t, \tilde{\phi})$ with an appropriate quadrature rule, and then using a semi-implicit method to approximate Eq. (11). After each $\delta^{k}$ is computed, it is added to $\tilde{\phi}$ to yield an updated provisional solution.

Specifically, (suppressing the $k$ index on $\delta$ ) a substep of the correction equation is

$$
\begin{aligned}
\delta^{m+1} & =\delta^{m}+\Delta t_{m}\left[F_{E}\left(t_{m}, \tilde{\phi}^{m}+\delta^{m}\right)-F_{E}\left(t_{m}, \tilde{\phi}^{m}\right)\right. \\
& \left.+F_{I}\left(t_{m+1}, \tilde{\phi}^{m+1}+\delta^{m+1}\right)-F_{I}\left(t_{m+1}, \tilde{\phi}^{m+1}\right)\right] \\
& +I_{m}^{m+1}(\tilde{\phi})-\tilde{\phi}^{m+1}+\tilde{\phi}^{m}
\end{aligned}
$$

where

$$
I_{m}^{m+1}(\tilde{\phi}) \approx \int_{t_{m}}^{t_{m+1}} F(\tau, \tilde{\phi}(\tau)) d \tau .
$$

As in [Min01], the points $t_{m}$ above are chosen to be the nodes of the standard Gauss-Labatto quadrature. Since the quadrature must be done for each subinterval $\left[t_{m}, t_{m+1}\right]$, there are actually $p$ quadrature rules of the form

$$
I_{m}^{m+1}(\tilde{\phi})=\sum_{l=0}^{p} q_{l}^{m} F\left(t_{l}, \tilde{\phi}^{l}\right)
$$

for $m=0 \ldots p-1$.

To summarize, a complete SISDC time step consists of the computation of the first provisional solution $\tilde{\phi}$ using $p$ substeps of Eq. (10) followed by $K$ iterations of the correction Eq. (12), each of which also involves $p$ substeps. It can be shown that for $K$ iterations of the correction equation, the above procedure will produce an approximate solution with overall error $O\left(\Delta t^{K+1}\right)$, provided the integrals in Eq. (13) have $O\left(\Delta t^{K+1}\right.$ ) error as well. (See [Min01] for a further discussion of accuracy and the choice of integration nodes.) 


\subsection{Stability of SISDC Methods}

To study the stability of the SISDC method for ODEs, it is most useful to consider the model problem

$$
\phi^{\prime}(t)=\lambda \phi=\alpha i \phi+\beta \phi .
$$

For the SISDC method, $F_{E}(t, \phi)=\alpha i \phi$ and $F_{I}(t, \phi)=\beta \phi$. This is the relevant model problem when studying the stability of finite difference methods for advection-diffusion type PDEs with $\alpha$ corresponding to the advection term and $\beta$ corresponding to the stiff diffusion term. For a given $\lambda$, let $\phi^{1}(\lambda)$ denote the result of taking one time step with the SISDC method applied to the model problem with $\phi(0)=1$ and $\Delta t=1$. The stability region is then defined as the set of $\lambda$ for which $\left|\phi^{1}(\lambda)\right|<1$.

Fig. 1 shows two stability regions for two different SISDC methods, both of which use three iterations of the correction equation (i.e. $K=$ 3 ). The larger region, however, corresponds to using four substeps per time step while the smaller only three. Both versions are fourthorder accurate but as explained in Sect. 4, projection methods based on the SISDC method require that four substeps be completed in order to compute the pressure to fourth-order accuracy. Although the stability region for this version is larger, a total of 16 implicit solves are required as opposed to only 12 for the version with only 3 substeps. The stability diagrams clearly show that the time step is restricted solely by the explicit term in the equation. See [Min01] for more information on the relative size of stability diagrams for SISDC methods.

\section{Projection Methods}

To help set the stage for the introduction of the semi-implicit projection methods in the next section, a brief discussion of first-order semi-implicit projection methods is now presented. For the moment, assume that the velocity and pressure have been discretized in time and denote by $\mathbf{u}^{n}$ the approximation to the velocity at time $t_{n}$ (and likewise $p^{n}$ ). For further ease of presentation an unbounded domain is considered. Boundary conditions are briefly discussed in Sect. 4.2. 


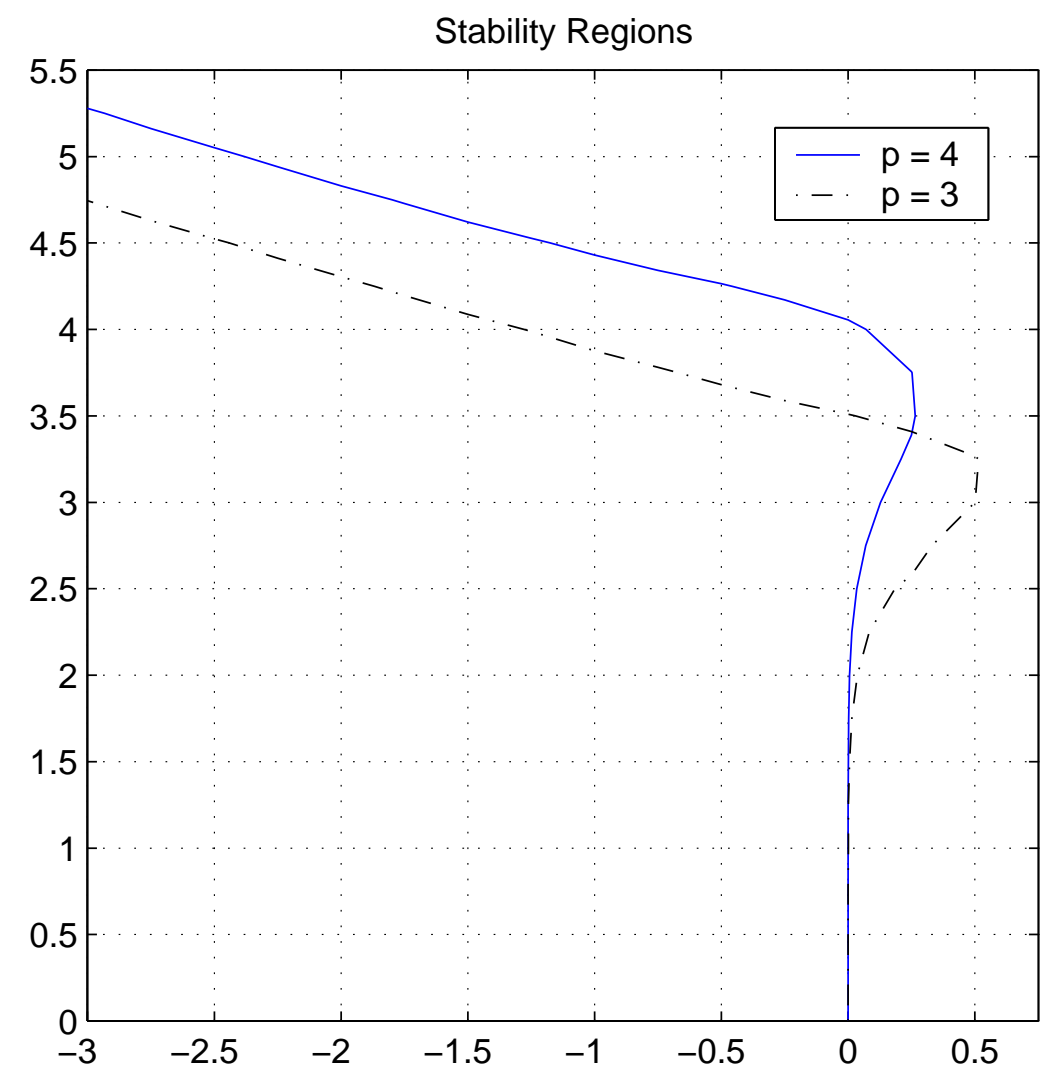

Figure 1: Stability regions for fourth-order SISDC methods. The two regions correspond to versions with $p=3$ substeps per time step and $p=4$ substeps. 
A first-order semi-implicit temporal discretization of Eq. (4) is

$$
\frac{\mathbf{u}^{n+1}-\mathbf{u}^{n}}{\Delta t}=\mathbf{P}\left[-\left(\mathbf{u}^{n} \cdot \nabla\right) \mathbf{u}^{n}-\nabla p^{n}+\nu \nabla^{2} \mathbf{u}^{n+1}\right] .
$$

Under many circumstances (depending on the boundary conditions that projected fields are required to satisfy), the pressure gradient term is annihilated by the projection and Eq. (14) is equivalent to

$$
\frac{\mathbf{u}^{n+1}-\mathbf{u}^{n}}{\Delta t}=\mathbf{P}\left[-\left(\mathbf{u}^{n} \cdot \nabla\right) \mathbf{u}^{n}+\nu \nabla^{2} \mathbf{u}^{n+1}\right] .
$$

Because of the difficulty in solving either of the above equations directly, usually an approximate two-step procedure is used instead. Specifically for Eq. (14), let $\mathbf{u}^{*}$ and $\mathbf{u}^{n+1}$ be defined by

$$
\begin{aligned}
\frac{\mathbf{u}^{*}-\mathbf{u}^{n}}{\Delta t} & =-\left(\mathbf{u}^{n} \cdot \nabla\right) \mathbf{u}^{n}-\nabla p^{n}+\nu \nabla^{2} \mathbf{u}^{*} \\
\mathbf{u}^{n+1} & =\mathbf{P}\left(\mathbf{u}^{*}\right) .
\end{aligned}
$$

For Eq. (15), the first of these two equations becomes

$$
\frac{\mathbf{u}^{*}-\mathbf{u}^{n}}{\Delta t}=-\left(\mathbf{u}^{n} \cdot \nabla\right) \mathbf{u}^{n}+\nu \nabla^{2} \mathbf{u}^{*}
$$

The term fractional step method is often used interchangeably with the term projection method to describe such a two step procedure. Note however, that by equating $\mathbf{u}^{*}$ with $\mathbf{m}^{n+1}$ where $\mathbf{m}$ is defined by Eq. (6), Eqs. (16) and (17) are also a first-order semi-implicit temporal discretization of Eqs. (6) and (8). To approximate Eq. (16), $\nabla q$ should be set to $\nabla p^{n}$. while on the other hand, $\nabla q=0$ for Eq. (18). In this sense, the projection methods above are not fractional step methods, and higher order semi-implicit projection methods can be constructed by considering the equations for $\mathbf{m}$ directly. Furthermore, the question of imposing boundary conditions for the intermediate quantity $\mathbf{u}^{*}$ is reduced to the question of imposing boundary conditions for $\mathbf{m}$. As mentioned above, there is a coupling between the boundary conditions for $\mathbf{m}$ and $\nabla \chi$ which must be respected in a numerical implementation in order to enforce the specified boundary conditions on $\mathbf{u}$.

\subsection{SISDC Projection Methods}

To construct fourth-order SISDC methods for the equations of incompressible flow, a method of lines approach is applied to Eq. (6). In the 
notation from Sect. $3, \phi \equiv \mathbf{m}$ while

$$
\begin{aligned}
F_{E} & =-(\mathbf{u} \cdot \nabla) \mathbf{u}-\nabla q \\
F_{I} & =\nu \nabla^{2} \mathbf{m} .
\end{aligned}
$$

To be more specific, the complete forward-backward Euler substep for computing the provisional solution in the SISDC method is

$$
\begin{aligned}
\tilde{\mathbf{m}}^{m+1} & =\tilde{\mathbf{m}}^{m}+\Delta t\left(-[(\tilde{\mathbf{u}} \cdot \nabla) \tilde{\mathbf{u}}]^{m}-\nabla q+\nu \nabla^{2} \tilde{\mathbf{m}}^{m+1}\right) \\
\tilde{\mathbf{u}}^{m+1} & =\mathbf{P}\left(\tilde{\mathbf{m}}^{m+1}\right) .
\end{aligned}
$$

All spatial derivatives are approximated with standard fourth-order centered-difference stencils. For each method below, $\mathbf{m}$ and $\mathbf{u}$ are evolved according to Eqs. (19-20) to yield the first approximate solution in the SISDC method. Three variants of this approach will be presented which differ in the choice of $\mathbf{m}, \nabla \chi$ and $\nabla q$. For all the methods, the term $\nabla q$ is held constant during the entire time step. One effect of this is that $\nabla q$ does not appear in the analog to Eq. (11). Letting $\hat{\mathbf{m}}=\tilde{\mathbf{m}}+\delta \mathbf{m}$ and $\hat{\mathbf{u}}=\tilde{\mathbf{u}}+\delta \mathbf{u}$, and rearranging Eq. (11) gives

$$
\begin{aligned}
\hat{\mathbf{m}}^{m+1} & =\hat{\mathbf{m}}^{m}+\Delta t\left[-\left(\hat{\mathbf{u}}^{m} \cdot \nabla\right) \hat{\mathbf{u}}^{m}+\left(\tilde{\mathbf{u}}^{m} \cdot \nabla\right) \tilde{\mathbf{u}}^{m}\right. \\
& \left.+\nu \nabla^{2} \hat{\mathbf{m}}^{m+1}-\nu \nabla^{2} \tilde{\mathbf{m}}^{m+1}\right]+I_{m}^{m+1}(\tilde{\mathbf{m}}) \\
\hat{\mathbf{u}}^{m+1} & =\mathbf{P}\left(\hat{\mathbf{m}}^{m+1}\right),
\end{aligned}
$$

where $I_{m}^{m+1}$ is defined in Eq. (13). In each variation, the new pressure term is computed by a finite-difference approximation of Eq. (9) after the full time step is completed. Since the temporal derivative term in Eq. (9) is not centered in time, five values of $\chi_{m}$ are needed to achieve fourth-order accuracy (i.e. $\mathrm{p}=4$ ). However, since three iterations of the correction equation are performed, the overall accuracy of the method is still order four.

The first variation of the SISDC projection method will be referred to as the "BCM" method since it is similar in spirit to the projection method introduced in [BCM01]. At the beginning of each time step in this variation, $\mathbf{m}^{0}$ is reset to $\mathbf{u}^{n}$, and $\nabla q$ is set to $\nabla p^{n}$. As mentioned above, $\nabla q$ is held constant during the time step, so the pressure term does not appear in the correction equation.

The second method will be referred to as the "KM" method since it is similar in spirit to the projection method introduced in [KM85]. As before, $\mathbf{m}^{0}$ is reset to $\mathbf{u}^{n}$ at the beginning of each time step, however 
$\nabla q$ is identically zero throughout the time step. Hence the KM method is a so-called pressure free method. This also eliminates the $\nabla q$ term in the computation of the pressure by Eq. (9).

The last method will be referred to as the "EL" method since it is similar in spirit to the methods introduced in [EL96, EL97]. In this variation, $\mathbf{m}$ is never reset to $\mathbf{u}$, hence in time the difference between $\mathbf{m}$ and $\mathbf{u}$ (i.e. $\nabla \chi$ ) can grow to have magnitude $O(1)$. As in the KM method $\nabla q$ is identically zero throughout the time step.

If one adopts a slightly different definition of what constitutes a projection method, i.e. a numerical method in which the velocity is obtained by the projection of an auxiliary variable, then all three of the preceding numerical methods can be thought of as projection methods. The only difference then between the three is the procedure used to advance the auxiliary variable. In this remainder of this paper, the term projection method will be used in this somewhat looser sense.

\subsection{Boundary conditions for semi-implicit pro- jection methods}

The emphasis in the current work is on the construction of higherorder semi-implicit time marching schemes and the relationship between the form of the evolution equation for the velocity and the pressure update equation. For the most part, the subject of numerical boundary conditions has been deferred.

It is important to point out that accurately imposing prescribed slip or in-flow boundary conditions for these methods is by no means a trivial matter. In [Min01] it is shown that even for a simple PDE such as the heat equation, using the prescribed boundary conditions for the PDE as the boundary conditions for the implicit step in the SISDC method will cause a degradation of accuracy similar to that which occurs with Runge-Kutta methods [SSVH86, CGAD95].

The situation is more complicated for projection methods where boundary conditions for $\mathbf{m}$ cannot be fully prescribed a priori. As is the case with the second-order semi-implicit methods described in [BCM01], the form of the evolution equation for $\mathbf{m}$ has a direct implication on how accurately the coupling of the boundary conditions for $\mathbf{m}$ and $\mathbf{u}$ must be approximated. In the present context, the implication is that the closer $\nabla q$ approximates the pressure gradient, the closer the boundary conditions for $\mathbf{m}$ will be to the prescribed condi- 
tions on u. A paper presenting specific details of imposing accurate boundary conditions for SISDC projection methods is in preparation.

\section{Convergence Studies}

To demonstrate the temporal accuracy of the SISDC projection methods, two numerical examples set in the simplified domain of the doublyperiodic unit square are considered. This removes the issue of imposing boundary conditions and allows the solution of the implicit equations by using the FFT. In the first example, the initial conditions are chosen so that the exact solution is known and convergence rates are calculated to corroborate the expected values. In the second example, the SISDC method is applied to the well studied problem of the roll-up of shear layers in a doubly-periodic geometry.

\subsection{Exact Solution}

For the first example, the projection methods are tested on the wellknown travelling wave solution to the Navier-Stokes equations. Specifically,

$$
\begin{aligned}
& u(x, y, t)=0.75+0.25 \cos (2 \pi(x-t)) \sin (2 \pi(y-t)) e^{-8 \pi^{2} \nu t} \\
& v(x, y, t)=0.75-0.25 \sin (2 \pi(x-t)) \cos (2 \pi(y-t)) e^{-8 \pi^{2} \nu t} \\
& p(x, y, t)=-\frac{1}{64}(\cos (4 \pi(x-t))-\cos (4 \pi(y-t))) e^{-16 \pi^{2} \nu t}
\end{aligned}
$$

The viscosity is set to $\nu=0.1$, and the time step is chosen to be $\Delta t=8.0 \Delta x$. For each method, errors are computed at time 0.5 in the $L_{\infty}$ norm for the pressure and $u$-component of the velocity for runs on grids of size $N \times N$ for $N=64,128$, and 256. Note that for the $256 \times 256$ runs, $\nu \Delta t / \Delta x^{2}=204.8$. Convergence rates calculated from the finest two runs are listed in Table 1 and are indicative of fourth-order convergence.

To be certain that the convergence rates in the above numerical result reflect temporal accuracy as opposed to spatial accuracy, the same example is repeated using a slightly different SISDC method. In this case, only 3 sub-steps are taken within each iteration, but 3 iterations of the correction equation are performed. This means that only 4 values of $\chi$ are available at the end of each time-step 


\begin{tabular}{|c|c|c|c|c|c|}
\hline \multicolumn{6}{|c|}{ Errors for exact solution problem } \\
\hline \multicolumn{2}{|c|}{} & $64 \times 64$ & $128 \times 128$ & $256 \times 256$ & rate \\
\hline BCM & $\mathbf{u}$ & $3.349 \mathrm{e}-5$ & $1.966 \mathrm{e}-6$ & $1.384 \mathrm{e}-7$ & 3.83 \\
\cline { 2 - 6 } & $p$ & $6.508 \mathrm{e}-7$ & $2.037 \mathrm{e}-8$ & $9.266 \mathrm{e}-10$ & 4.46 \\
\hline \hline \multirow{2}{*}{ KM } & $\mathbf{u}$ & $3.349 \mathrm{e}-5$ & $1.966 \mathrm{e}-6$ & $1.384 \mathrm{e}-7$ & 3.83 \\
\cline { 2 - 6 } & $p$ & $6.970 \mathrm{e}-7$ & $2.092 \mathrm{e}-8$ & $9.031 \mathrm{e}-10$ & 4.53 \\
\hline \hline \multirow{2}{*}{$\mathrm{EL}$} & $\mathbf{u}$ & $3.349 \mathrm{e}-5$ & $1.966 \mathrm{e}-6$ & $1.384 \mathrm{e}-7$ & 3.83 \\
\cline { 2 - 6 } & $p$ & $4.097 \mathrm{e}-7$ & $1.946 \mathrm{e}-8$ & $1.084 \mathrm{e}-9$ & 4.16 \\
\hline
\end{tabular}

Table 1: $L_{\infty}$ errors for the $u$-component of velocity and the pressure for the exact solution test problem. The rates are computed from the errors in the $128 \times 128$ and $256 \times 256$ grids.

\begin{tabular}{|l|c|c|c|c|}
\hline \multicolumn{5}{|c|}{ Errors in the pressure } \\
\hline & $64 \times 64$ & $128 \times 128$ & $256 \times 256$ & rate \\
\hline BCM & $1.963 \mathrm{e}-6$ & $2.036 \mathrm{e}-7$ & $2.246 \mathrm{e}-8$ & 3.18 \\
\hline KM & $2.816 \mathrm{e}-6$ & $2.483 \mathrm{e}-7$ & $2.624 \mathrm{e}-8$ & 3.24 \\
\hline EL & $2.704 \mathrm{e}-6$ & $2.714 \mathrm{e}-7$ & $2.848 \mathrm{e}-8$ & 3.25 \\
\hline
\end{tabular}

Table 2: $L_{\infty}$ errors for the pressure for the exact solution test problem with only 3 substeps per time step. The rates are computed from the errors in the $128 \times 128$ and $256 \times 256$ grids and reflect the fact that the temporal part of the pressure equation is being computed with only third-order accuracy.

with which to calculate the time derivative piece of the pressure in Eq. (9). Therefore, if temporal error is significant the accuracy of the pressure should drop to third order. The errors and convergence rates for this example are shown in Table 2 and demonstrate thirdorder accuracy. The errors for the velocity are not shown but remain $O\left(\Delta t^{4}\right)$ as expected.

\subsection{Double Shear Layer}

The second numerical example is performed on an unsteady flow within the same geometry and given by the perturbed shear layer initial conditions

$$
u= \begin{cases}\tanh (30(y-0.25)), & \text { for } y \leq 0.5 \\ \tanh (30(0.75-y)), & \text { for } y>0.5\end{cases}
$$




\begin{tabular}{|c|c|c|c|c|c|}
\hline \multicolumn{6}{|c|}{ Errors for the shear layer problem } \\
\hline \multicolumn{2}{|c|}{} & $64 \times 64$ & $128 \times 128$ & $256 \times 256$ & rate \\
\hline \multirow{2}{*}{ BCM } & $\mathbf{u}$ & $8.311 \mathrm{e}-4$ & $5.544 \mathrm{e}-5$ & $3.477 \mathrm{e}-6$ & 4.00 \\
\cline { 2 - 6 } & $p$ & $3.887 \mathrm{e}-4$ & $2.576 \mathrm{e}-5$ & $1.628 \mathrm{e}-6$ & 3.98 \\
\hline \hline \multirow{2}{*}{ KM } & $\mathbf{u}$ & $1.158 \mathrm{e}-3$ & $8.000 \mathrm{e}-5$ & $4.296 \mathrm{e}-6$ & 4.22 \\
\cline { 2 - 6 } & $p$ & $1.185 \mathrm{e}-3$ & $5.395 \mathrm{e}-5$ & $2.662 \mathrm{e}-6$ & 4.34 \\
\hline \hline \multirow{2}{*}{$\mathrm{EL}$} & $\mathbf{u}$ & $3.816 \mathrm{e}-3$ & $2.520 \mathrm{e}-4$ & $1.574 \mathrm{e}-5$ & 4.00 \\
\cline { 2 - 6 } & $p$ & $3.892 \mathrm{e}-3$ & $2.543 \mathrm{e}-4$ & $1.588 \mathrm{e}-5$ & 4.00 \\
\hline
\end{tabular}

Table 3: Errors for the pressure and $u$-component of the velocity for the shear layer test problem. The rates are computed from the errors in the $128 \times 128$ and $256 \times 256$ grids by comparison with the reference solutions.

$$
v=0.05 \sin (2 \pi(x+0.25)) \text {. }
$$

The viscosity is set to $\nu=0.002$ for all runs. The exact solution for this problem is not known, therefore for each method, a reference solution on a $768 \times 768$ grid using $\Delta t=2 \Delta x$ is used as an exact solution. Three numerical runs using grids of size $64 \times 64,128 \times 128$, and $256 \times 256$ and with $\Delta t=4 \Delta x$ are used to estimate convergence rates. Errors are calculated in the $L_{\infty}$ norm at $t=1.0$ by comparison with the reference solution. Convergence results for the velocity and the pressure shown in Table 3 again indicate fourth-order convergence.

The parameters for this example are the same as those used in a convergence study in [MB97] where the velocity computed by a spectral collocation method was used as a reference solution. (The spectral method does not compute the pressure.) The velocities from the reference solutions computed by the SISDC differ from this reference solution by an amount that is at least two orders of magnitude smaller than the errors used to compute the convergence rates in Table 3 . This direct comparison leaves little doubt that the projection methods above are indeed converging to the exact solution at a fourth-order rate.

\section{Conclusion}

In this paper, a general strategy for constructing semi-implicit projection methods for the incompressible Navier-Stokes equations with 
higher-order temporal accuracy is presented. The strategy utilizes recently introduced semi-implicit methods for ODEs based on spectral deferred corrections. Three variations of a generalized projection method are presented and fourth-order accuracy is demonstrated on flows in a two-dimensional doubly-periodic domain.

In terms of implicit equations that need to be solved, the projection methods in this paper require only the solution of the Poisson equation associated with the projection and the implicit equation in each timestep which takes the form

$$
\left(I-\nu \Delta t \nabla^{2}\right) u=f .
$$

Both of these equations are linear, and higher-order solvers have been developed for each based on using the Fast Multipole Method to efficiently compute the convolution integral solution to the equation. For the two-dimensional Poisson equation, adaptive methods suitable for use with irregular domains have recently been implemented [EG00] that incorporate standard block rectangular mesh refinement (see e.g. [BO84]) and classical layer potentials for imposing boundary conditions. Versions with up to eighth-order spatial accuracy have been developed, and the computational cost per grid point of these methods is only a small factor times that for a uniform grid FFT-based solver. The analogous algorithms for Eq. (21) in a two-dimensional rectangular geometry have also been implemented [HGE01], and the complex geometry versions are currently being developed. A project to incorporate these solvers into projection methods for two-dimensional incompressible flow in complex geometry is also underway. Three dimensional versions of all the algorithms have been described, but the implementation is still in the future.

It is worth reiterating that despite the similarities of the three methods described in this paper and the comparable performance on the test problems presented, the issues concerning accurately imposing prescribed slip or in-flow boundary conditions differ in a significant way between variations. A paper describing in detail these issues is in preparation.

\section{References}

[AGC96] Saul Abarbanel, David Gottlieb, and Mark H. Carpenter. On the removal of boundary errors caused by Runge-

This work was performed under the auspices of the United States Department of Energy by the University of California, Lawrence Livermore National Laboratory under contract No. W-7405-Eng-48. 
Kutta integration of nonlinear partial differential equations. SIAM J. Sci. Comput., 17:777-782, 1996.

[ARS97] Uri M. Ascher, Steven J. Ruuth, and Raymond J. Spiteri. Implicit-explicit Runge-Kutta methods for timedependent partial differential equations. Appl. Num. Math., 25:151-167, 1997.

[ARW95] Uri M. Ascher, Steven J. Ruuth, and Brian T. R. Wetton. Implicit-explicit methods for time-dependent PDE's. SIAM J. of Numer. Anal., 32:797-823, 1995.

[BCG89] J. B. Bell, P. Colella, and H. M. Glaz. A second order projection method for the incompressible Navier-Stokes equations. J. Comput. Phys., 85(2):257-283, December 1989.

[BCM01] D. L. Brown, R. Cortez, and M. Minion. Accurate projection methods for the incompressible Navier-Stokes equations. J. Comput. Phys., 168, Apr. 2001.

[BO84] M. J. Berger and J. Oliger. Adaptive mesh refinement for hyperbolic partial differential equations. J. Comput. Phys., 53:484-512, 1984.

[But93] T. Buttke. Velicity methods: Lagrangian numerical methods which preserve the Hamiltonian structure of incompressible fluid flow, pages 39-57. Kluwer Academic Publisher, 1993. NATO ASI Series C, vol. 395.

[CdFN01] M. P. Calvo, J. de Frutos, and J. Novo. Linearly implicit Runge-Kutta methods for the advection-reaction-diffusion equations. Appl. Num. Math., 37:535-549, 2001.

[CGAD95] Mark H. Carpenter, David Gottlieb, Saul Abarbanel, and Wai-Sun Don. The theoretical accuracy of Runge-Kutta time discretization for the initial boundary value problem: A study of the boundary error. SIAM J. Sci. Comput., 16:1241-1252, 1995.

[Cho68] A. J. Chorin. Numerical solution of the Navier-Stokes equations. Math. Comp., 22:742-762, 1968.

[Cho69] A. J. Chorin. On the convergence of discrete approximations to the Navier-Stokes equations. Math. Comp., 23:341-353, 1969. 
[CM00] Ricardo Cortez and Michael L. Minion. The blob projection method for immersed boundary problems. J. Comput. Phys., 161:428-453, 2000.

[Cor95] Ricardo Cortez. Impulse-based Particle Methods for Fluid Flow. PhD thesis, University of California, Berkeley, May 1995.

[Cor96] Ricardo Cortez. An impulse-based approximation of fluid motion due to boundary forces. J. Comput. Phys., 123:341-353, 1996.

[CP01] M. P. Calvo and C. Palencia. Avoiding the order reduction of Runge-Kutta methods for linear initial boundary value problems. Math. Comp., 2001. to appear.

[DGR00] Alok Dutt, Leslie Greengard, and Vladimir Rokhlin. Spectral deferred correction methods for ordinary differential equations. BIT, 40(2):241-266, 2000.

[EG00] F. Ethridge and L. Greengard. A new fast-multipole accelerated Poisson solver in two dimensions. 2000. submitted.

[EL96] Wienan E and Jian-Guo Liu. Gauge method for viscous incompressible flows. Unpublished, 1996.

[EL97] Wienan E and Jian-Guo Liu. Finite difference schemes for incompressible flows in the velocity-impulse density formulation. J. Comput. Phys., 130:67-76, 1997.

[FHV97] J. Frank, W. H. Hundsdorder, and J. G. Verwer. Stability of implicit-explicit linear multistep methods. Appl. Num. Math., 25:193-205, 1997.

[HGE01] J. Huang, L. Greengard, and F. Ethridge. A new fastmultipole accelerated Yukawa solver in two dimensions. 2001. In preparation.

[HRSZ99] T. Hagstrom, K. Radhakrishnan, S. Steinberg, and R. Zhou. Simulation of unsteady combustion phenomena using complex models. In 35th AIAA/ASME/SAE/ASEE Joint propulsion conference and exhibit, June 1999.

[HRZ98] T. Hagstrom, K Radhakrishnan, and R. Zhou. Computation of steady and unsteady laminar flames: theory. In 34th AIAA/ASME/SAE/ASEE Joint propulsion conference and exhibit, July 1998. 
[KC01] Christopher A. Kennedy and Mark H. Carpenter. Additive Runge-Kutta schemes for convection-diffusion-reaction equations. Appl. Num. Math., 2001. Submitted.

[KM85] J. Kim and P. Moin. Application of a fractional-step method to incompressible Navier-Stokes equations. $J$. Comput. Phys., 59:308-323, 1985.

[MB97] M. L. Minion and D. L. Brown. Performance of underresolved two-dimensional incompressible flow simulations II. J. Comput. Phys., 138:734-765, 1997.

[Min01] M. L. Minion. Higher-order semi-implicit methods for initial boundary value partial differential equations. In preparation.

[Ose89] V. I. Oseledets. On a new way of writing the Navier-Stokes equation: The Hamiltonian formalism. Commun. Moscow Math.Journal of Society, 44(1):210-211, 1989.

[Pat97] D. Pathria. The correct formulation of intermediate boundary condidtions for Runge-Kutta time integration of intitial boundary value problems. SIAM J. Sci. Comput., 18(5):1255-1266, 1997.

[RR98] M. C. Recchioni and G. Russo. Hamiltonian-based numerical methods for a fluid-membrane interaction in two and three dimensions. SIAM J. Sci. Comput., 19:861-892, 1998.

[SC96] D. M. Summers and A. J. Chorin. Numerical vorticity creation based on impulse conservation. Proc. Nat. Acad. Sci. USA, 93:1881-1885, 1996.

[SSVH86] J. M. Sans-Serna, J. G. Verwer, and W. H. Hundsdorfer. Convergence and order reduction of Runge-Kutta schemes applied to evolutionary problems in partial differential equations. Num. Math., 50:405-418, 1986.

[SZ96] J. W. Shen and X. Zhong. Semi-implicit Runge-Kutta schemes for the non-autonomous differential equations in reactive flow computations. In Proceedings of the 27th AIA A Fluid Dynamics Conference. AIAA, June 1996. 